\title{
Assessing Coverage of Targeted and Large-scale Fortification Programs: Development of a Fortification Assessment Coverage Tool (FACT)
}

\author{
Grant Aaron ${ }^{1 \star}$, Katja Siling $^{2}$, Allie Norris ${ }^{2}$, Ernest Guevarra ${ }^{2}$ and Mark Myatt ${ }^{3}$ \\ ${ }^{1}$ Global Alliance for Improved Nutrition (GAIN), Geneva, Switzerland. \\ ${ }^{2}$ Valid International, Oxford, UK. \\ ${ }^{3}$ Brixton Health, Wales, UK.
}

Article Information

DOI: $10.9734 / E J N F S / 2015 / 21171$

Special Editors:

Lucie Bohac, Micronutrient Forum Secretariat, Canada. Klaus Kraemer, Director, Sight and Life, Basel, Switzerland.

Chief Editor

Prof. Hans Verhagen, Senior Scientific Advisor 'Nutrition and Food Safety', National Institute for Public Health and the Environment (RIVM), P.O.Box 1, 3720 BA, Bilthoven, The Netherlands.

Objectives: To develop and operationalize a survey tool that can be used by nutrition programmers to assess and classify coverage and consumption patterns in targeted (point-of-use) and large-scale (staple) fortification programs.

Methods: We focused on developing a tool that is low-cost, and rapid for programs to implement, analyze, and assess coverage and barriers to coverage during the program cycle. Indicators were adapted from validated guidelines, and subject matter experts were consulted. We emphasize simple spatial survey (S3M) methods, which allow for fine scale geographical coverage maps and wide-area population-level estimates to be produced. The FACT tool can also be implemented using cluster survey methods.

Results: A pilot FACT survey using S3M methods was first undertaken in three administrative districts in Ghana to refine the survey methods and instruments. Results were reported back to the program within two weeks of survey completion to provide a quick feedback loop to guide programming efforts. Subsequent implementation of the FACT tool has been applied to a statewide S3M survey in Rajasthan, India, and National cluster survey in Senegal.

Conclusions: We have developed and operationalized a tool that can be applied to coverage assessments of targeted and large-scale fortification programs. Further surveys assessing coverage in programs supported by The Global Alliance for Improved Nutrition (GAIN) programs 
are planned. Additional work on the FACT tool will include refining the methods and instruments after running additional survey rounds, refining analysis and reporting tools, and disseminating in the public domain.

(c) 2015 Aaron et al.; This is an Open Access article distributed under the terms of the Creative Commons Attribution License (http://creativecommons.org/licenses/by/4.0), which permits unrestricted use, distribution, and reproduction in any medium, provided the original work is properly cited. 